\title{
ARCHITECTURAL HYPER-MODEL: CHANGING ARCHITECTURAL CONSTRUCTION DOCUMENTATION
}

\author{
ANTONY PELOSI \\ Spatial Design, Institute of Design \\ Massey University, Wellington \\ PO Box 576, Wellington, 6001, New Zealand \\ a.pelosi@massey.ac.nz
}

\begin{abstract}
More architects and spatial designers are producing complex 3D computer models as part of their everyday design process and documentation than ever before. Parallel to this shift, there has been a rapid rise in consumer computer processing power that has made hyper realistic digital environments a part of our home entertainment. Together, the 3D CAD models and the Computer Gaming Engine could become an architectural hyper-model that renders a digital environment in real time. Such a model would enable users to navigate freely, effectively establishing a new mode of reading space that hovers between 2D drawings and a real space.(Nitsche \& Roudavski). This paper will examine how these worlds can merge to form an architectural hyper-model as a valuable supplement to the more conventional scaled 2D construction drawing documentation found on construction sites.

While easily misconstrued as speculative, the ideas presented in this paper outline an on-going body of innovative research currently at the prototype stage. These prototypical hyper-models explore the possibilities of providing construction workers and project mangers access to an architect's 3D computer models on site. These models originate from within conventional building construction drawings such as detailed sections and exploded axonometrics. A process of reinterpretation occurs to locate these drawings and their information within an interactive 3D space. Such operations take advantage of the best of both paradigms. This gives users access to, and control of, the 3D information required for communicating necessary information about the building process. It also provides nodes or hyper-links in the 3D representation that connect to additional information, such as specifications, that are perhaps less formal/spatial.

The paper will show how architectural hyper-models can be used on the construction site - both in the site office and on site using laptop computers and more compact hand-held devices - to decrease on site confusion and enable a faster and more complete understanding of the architect's vision. The paper concludes with speculation on the types of additional information construction workers, architects and designers might want to access in the future and proposes additional technologies that could be provided.
\end{abstract}

Keywords: architectural hyper-model, construction drawings, documentation technology, entertainment, construction visualization. 


\section{Introduction}

Historically, architects have produced a set of 2D drawings that have abstract information spread across different scales and views. Coupled with the fact that a set of drawings is typically multiple sheets, the proposed building project is difficult to visualize as a complete object and process of construction. Contractors have to piece together information within 2D drawings, which has been collected and packed by architects. This form of communicating has been the established best practice for over 500 years.

Wakita and Linde points to the powerful way computers are being used to create digital 3D models when they comment that we now draw buildings in such a fashion that the computer monitor becomes a type of window through which we are able to view full-size buildings in space. Buildings are drawn in 3-D and rotated into plan and elevation, or rotated and sliced to produce sections, framing and floor plans. This rotation and slicing process helps the architectural technician and student better understand what the construction documents entail. (vii)

The opening paragraph from Gehry Technologies corporate brochure outlines the requirements of a modern construction documentation delivery system: Building projects are increasingly complex undertakings. Tougher building codes and performance requirements, tighter schedules, distributed teams, and the possibility of new architectural forms, all add up to a building design and construction process whose demands exceed the capabilities of 2D CAD and paper based delivery processes. Additional complexity in the design phase has created downstream problems in construction where poor data coordination translates directly into an average of $20 \%$ costs overruns during construction. (1)

This research starts to investigate methods of providing non-paper based construction information to construction workers on site.

An architectural hyper-model provides a real time 3D architectural representation within a digitally rendered immersive environment that enables user navigation and interaction. This is achieved by utilizing current generation computer gaming software technology. The notion of hyper draws on two definitions: relating to hypertext or hyperlink, enabling one to hyperjump from place to place without having to visit points in between as noted by Kalay (467); and hyper, meaning over, above or beyond (current digital architectural models). Providing more control and content for the viewer. Currently most CAD programs work within an 'object' based mode, using legacy methods and concepts from the drawing board. An architect or designer constructs a building or object in digital space and then zooms in and out, rotating and pan-ing around it. This type of navigation loses any sense of scale, context and relationships between items. Gravity and sense of ground are also eliminated or suspended. These representation modes impede comprehension of the building or object.

Computer gaming software uses an 'environment' mode rather than an object mode to display architectural space, giving a viewer centric point or first person perspective view. The shift from object based modeling to environment based modeling changes the way the information can be viewed and understood, providing a scaled space which is navigated by walking or flying and hyper-linking in real time. The first person view gives a stronger understanding of scale and relationships of the proposed building. The current technology enables these spaces to be dynamic, items can be moveable, even picked up and changed, using real world physics.

This ability to create truly dynamic interactive environments enables the hyper-model to provide more than just a walk-through of the proposed design, it offers active links to additional content, such as drawings and specifications. It also allows for details to become truly connected to their location by being hyperlinked in space. In addition, items can be animated to provide construction sequence information, giving contractors a better understanding of the details they have to construct.

The key benefits of using an architectural hyper-model are that sections and details can be connected back to their location in the building through hyper linking, so items are not left floating. Other benefits of using an architectural hyper-model as a method of construction information communication include;

- Scalable view -- can be viewed on any sized screen without modification (desktop and handheld computers)

- Easy navigation control - based on standard computer gaming navigation

- Material identification - with the use of realistic and coded textures 
- Interactive - viewer controlled elements

- $\quad$ Real-time physics - ability to introduce 3D objects in the hyper-model, which react with real-world physical attributes.

- $\quad$ Multiples - unlike physical models a hyper-model can be viewed in multiple locations at once (only requiring additional hardware).

- Collaborative environment- real time meetings within the model

- Document management - The hyper-model would be stored online to ensure the latest model is accessed by all.

\section{Construction Visualization}

Transfer of information between participants in the design and construction process is facilitated by spatial visualizations, typically encoded 2D plan and section drawings and specifications. Acording to Saidi, et al "Construction is an information intensive industry in which the accuracy and timeliness of information is paramount" (1). Construction workers and contractors have expressed a desire to improve their visualization and interpretation of (decoding) construction drawings in order to understand what a designer has documented. It is critical for construction workers to fully understand the designer's construction documentation to reduce construction time and cost by reducing misunderstandings. Saidi, et al proposes, "The construction industry is in need of tools that can provide accurate, reliable, and timely project information to the field." (1)

Professor Kalay, Director of the UC Berkeley Center for New Media, states scale drawings provide a parsimonious notational means of conveying both referential and frame-of-reference (context) information, in the form of floor plans, sections, elevations, and details. However, much of the information that is conveyed by drawing is implicit and relies heavily on interpretation... This heavy reliance on interpretation, and the need to augment the explicit information with implicit assumptions, hinders the effective use of drawings as a means to engender shared understanding (481).

Now that architects are producing 3D computer models the problem is how to get this information on to the construction site. Gehry Technologies comments on "The potential for digital technologies to change the nature of professional practice and address the underlying inefficiencies and conflicts resulting from and outdated process". (2). Gehry Partners provide the contractor with a full 3D CAD model as part of the contract documentation. The CAD software is complex and requires an experienced operator. This leaves a gap between the CAD operator and the construction workers opening up another area for confusion and productivity loss.

In his book Architecture's New Media, Kalay notes that "The opening of a new kind of space made possible by computers and networks promises to revolutionize our perception of reality like no other invention before it and challenges the professions of architecture, town planning, and interior design which have been striving to accommodate human activities in the physical domain for thousands of years." (80)

A study carried out at the Pennsylvania State University using 4D CAD modeling in the education of architectural engineering program to teach construction processes suggests, "students can understand construction projects and plans much better when advanced visualization tools are used" (Messner 1). Johns and Lowe report similar findings with landscape architecture students at Victoria University of Wellington, "Four major benefits of using real-time modeling over physical scale modeling identified by the students were; comprehension of scale, engagement of other senses with sound, understanding space and time, and the ability to interact with others in a virtual landscape." (Johns and Lowe 1)

\section{3D computer models}

Architects and spatial designers are moving from 2D CAD software to 3D CAD software and creating complex detailed 3D computer models. How can these 3D computer models be used for more than the design process and marketing? It no longer makes practical sense to only use $2 \mathrm{D}$ drawings to communicate complex $3 \mathrm{D}$ space. Currently most designers end up converting the $3 \mathrm{D}$ models into 2D drawings for construction documentation, losing the benefits of the models; relationship of elements, context of spaces, control of view and layer control.

Presently, the most significant challenge resides in how to view not just 3D models on site, but any digital drawings. A number of recent research projects using handheld computer to access 
2D CAD drawings on site show little success due to the small screen real estate which hinder the display of drawings formatted for large paper sizes. The processing power required to access a full 3D CAD model is still limited to laptop computers, which are cumbersome and expensive to use on an actual construction site. As most current 3D models presented as rendered images or pre-rendered walk/fly through animations produced to explain the project to clients or for marketing material, have a fixed view, limiting the full potential of 3D CAD models. These factors make it time consuming to produce and limit the use of these images and animations on the construction site. Instead, a hyper-model renegotiates the relationship between architect and builder by assisting builders and contractors to envision the built work and its installation process more vividly. One of the major benefits of these models is to help contractors visualize what it is they are building.

\section{Experiments}

Two experiments by the author explore the interactive power of the 3D game engine Source ${ }^{\mathrm{TM}}$ by Valve Software Corporation through the use of the architectural drawing conventions of the section and exploded axonometric. Both experiments use Ludwig Mies van der Rohe's Barcelona Pavilion to demonstrate the potential of this technology. These experiments were carried out to investigate the potential application of current gaming engines for architectural visualization purposes. Both experiments are based on $2 \mathrm{D}$ drawing conventions and developed with the interactive possibilities that the real time 3D game engine provide.

The section pavilion (fig 1) explores the notion of representing a section and its accompanying information within an interactive real time 3D virtual environment, rather that just a flat $2 \mathrm{D}$ sheet of paper, which is isolated from its location and heavily encoded. Instead of the sections being referenced from a plan or other drawing in a customary manner, the sections within the pavilion hyper-model are identified by numbered markers indicating their location and upon selecting one of the thickened sections, it moves out from its actual location to sit next to the model.

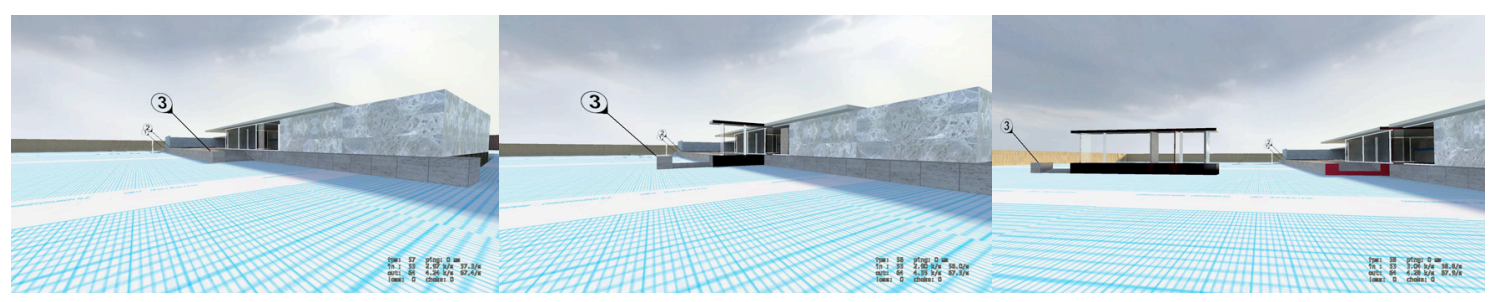

Figure 1: Still images from Section Pavilion hyper-model

The layer pavilion (fig 2) takes the exploded axonometric into the interactive environment giving a viewer the ability to control which parts of the Barcelona Pavilion are pulled up off the base, letting a viewer experience the separate elements and demonstrating the "essence of the Barcelona Pavilion. This mechanism facilitates analysis of the different building elements and the relationship between components.

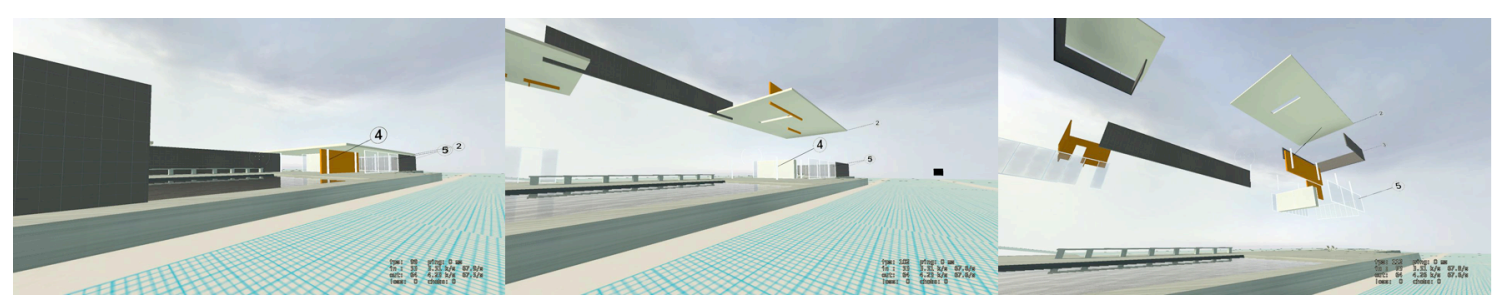

Figure 2: Still images from Layer Pavilion hyper-model

It becomes clear from these experiments that an architectural hyper-model provides useful methods of visualization, but can also introduce confusion as conventional drawing methods do. Therefore the research suggests another language needs to be developed that builds on the potential of the real time interactive power of the software. 


\section{The Hardware}

Desktop computers are commonplace in architects and construction site offices, but until recently they have only been used as a digital drawing board. Current CAD software packages work well for designers and architects, but the information is still being documented through 2D drawings. Currently considerable effort and time have been put in to the CAD software industry to enable easier and faster input and control, with little research focused on the delivery of construction documentation.

Handheld computers have been used on construction sites from as early as 1992, but have never been successful due to lack of processing power, screen size, and limited software. Recently handheld computers have increased in processing power and screen resolution which has led to a greater use on construction sites, speeding up communication, mostly for text based information as their has been no useful way to view construction drawings on their small screen (Saidi, et al 2). Intel estimates that, with current software, utilizing handheld and mobile computing on site would boost productivity by $20 \%$ to $30 \%$ (1)

One problem with handheld units is that the $2 \mathrm{D}$ drawings have been formatted for large sheets of paper. While some construction details are viewable on the small screen of a handheld computer, they become hard to reference from a large scale digital plan or section. By changing the method of describing space from just $2 \mathrm{D}$ drawings to dynamic navigable $3 \mathrm{D}$ space, most of these problems can be solved by:

- Being able to visualize the design proposal in real time

- Having access to construction documentation/3D models on site (PDA PSP)

- Collaborating and communicating in real time between multiple participants

- Virtual meetings in a shared, information enriched 3D online space

\section{Future directions}

The research so far points to additional technologies that can be added to the handheld computer providing greater functionality. RFID ${ }^{1}$ readers and global positioning system (GPS) modules represent potential additive technologies. In-depth analysis of these items exceeds the scope of this research; the following outlines only how they could be developed in the future.

It is predicted that RFID tags will replace barcodes in the coming years, enabling an architectural hyper-model running on a handheld computer with a RFID reader to access location and detail information about tagged items as they come within proximity of that item, all shown in real time and 3D. For example, as steel trusses are delivered on site, the construction worker can be shown the exact location each truss is to be installed So rather than searching through hundreds of drawings to find their location, the information is provided automatically.

In addition to this the architectural hyper-model could be fitted with a GPS module to provide location based information. As a construction worker or architect walked around the construction site with a handheld computer the GPS would locate them within the hyper-model. This would enable a clearer understanding of the location of the proposed building and its elements.

\section{Conclusion}

Presently paper based scaled drawings are the dominant means of construction visualization within the construction industry. As the use of computers and CAD software within the architectural design process has become standard, the development of the software has progressed along with the up-take of the software technology in architectural practice over the last 50 years. The only change we have seen in the construction documentation is an increase in the amount of information provided.

This research proposes to introduce architectural hyper-models with the aim of enhancing comprehension of construction drawings and specifications. By making the visualization of design proposals faster and clearer, the number of drawings required is reduced, ultimately increasing productivity and quality.

Moving away from an 'object' mode based CAD software to an 'environment' mode for the visualization provides easier access to the complex information required to build, as building projects are faced with complex requirements and tighter deadlines. The technology used allows the environments of the hyper-models to be truly dynamic; the geometry, location and visibility can be controlled providing more than just a walk-through of the project. 
The pavilion project clearly demonstrates the dynamic abilities of the hyper-model as an innovative method of explaining construction information. With the ability to scale from large desktop screens to small handheld computers the content does not need to be reformatted to give access to clients at home or laborers on site. As other technologies develop, such as RFID and GPS, these can be added to the architectural hyper-model providing greater function.

\section{Notes}

1 Radio-frequency identification (RFID) is an automatic identification method, relying on storing and remotely retrieving data using devices called RFID tags or transponders. An RFID tag is an object that can be attached to or incorporated into a product, animal, or person for the purpose of identification using radio waves. Chip-based RFID tags contain silicon chips and antennas. Passive tags require no internal power source, whereas active tags require a power source. (http://en.wikipedia.org/wiki/RFID).

\section{$9 \quad$ References}

Intel "Savings of 20 to 30 percent with Inte $\Theta^{\circledR}$ Centrino ${ }^{\mathrm{TM}}$ mobile technology help Laing O'Rourke build business" Intel Business Center Case Study. http://www.intel.com/ca/business/casestudies/pdf/laing_orourke.pdf (Accessed August 16, 2007)

Johns, Ralph, and Russell lowe. "Unreal Editor as a Virtual Design Instrument in Landscape Architecture Studio." Journal of Design Research 5.2 2006: 172-87.

Kalay, Yehuda E. Architecture's New Media : Principles, Theories, and Methods of ComputerAided Design. Cambridge, Mass. ; London :: MIT, 2004.

Messner, John I., et al. "Using Virtual Reality to Improve Construction Engineering Education " American Society for Engineering Education Annual Conference \& Exposition. Nashville: American Society for Engineering Education 2003.

Nitsche, Michael, and Stanislav Roudavski. "Building Cuthbert Hall Virtual College as a Dramatically Engaging Environment." Participatory Design Conference. Eds. T.Binder, J.Gregory and I.Wagner. Malmoe, Sweden: CPSR, 2002. pp 386-89.

Saidi, K. S., C. T. Haas, and N. A Balli. "The Value of Handheld Computers in Construction." International Symposium on Automation and Robotics in Construction. Gaithersburg, Maryland, 2002. 557-62.

Wakita, Osamu A., and Richard M. Linde. The Professional Practice of Architectural Working Drawings 3rd ed. London : New York :: Wiley-Academy J. Wiley \& Sons, 2003. 